\title{
Tobacco and e-cigarette use, and exposure to SHS among health sciences students: a national cross-sectional study
}

Sofia Belo Ravara ${ }^{1}$, Vera Afreixo ${ }^{2}$, Mónica Condinho ${ }^{3}$, Marília Santos Rua ${ }^{4}$, Carlos Albuquerque $^{5,}$ Pedro Aguiar $^{6}$ and Jorge Bonito ${ }^{7}$

${ }^{1}$ Faculty of Health Sciences, University of Beira Interior; CHCB University Hospital, Covilhã; Public Health Research Centre (CISP), National School of Public Health, NOVA University, Lisbon, Covilha and Lisbon, Portugal

${ }^{2}$ Departamento de Matemática da Universidade de Aveiro, Aveiro, Portugal

${ }^{3}$ Faculdade de Ciências e Tecnologia da Universidade do Algarve; Acompanhamento Farmacoterapêutico, Lda, Faro, Portugal

${ }^{4}$ Escola Superior de Saúde e CIDTFF da Universidade de Aveiro, Aveiro, Portugal

${ }^{3}$ Escola Superior de Saúde \& CI\&DETS do Instituto Politécnico de Viseu, Viseu, Portugal

${ }^{6}$ Public Health Research Centre (CISP), National School of Public Health, NOVA University, Lisbon, Portugal

${ }^{7}$ Departamento de Pedagogia e Educação da Escola de Ciências Sociais, Universidade de Évora; CIDTFF da Universidade de Aveiro, Évora, Portugal

\section{Abstract}

Aim: To assess tobacco and e-cigarette (EC) use, and exposure to SHS among pharmaceutical (PHs), nursing (Ns), medical (MDs), and medical dental (Ds) finalists students.

Methods: In 2016, a national cross-sectional questionnaire-based study involved health science schools in Portugal (46.6\% online). A descriptive/inferential and regression analysis was performed.

Results: Participants: 2095 students, 79.5\% females, mode age 23 years, 34.5\% collaboration rate. Of the finalists, more than half had experimented tobacco (in descending order: box cigarettes, RYO, shisha, cigarillos, cigars, $\mathrm{p}<0.001)$. Experimentation of $E C$ ranged from $9.3 \%$ (MD) to $13.4 \%$ (Ns). EC occasional consumption was more frequent than daily. Prevalence of tobacco use was: Ns-22.1\% (23.5\% in males; $21.8 \%$ in females, $p=0.6)$; Ds- $19.7 \%$ (29.7\% in males; $15.9 \%$ in females, $p=0,018)$; PHs19.4\% (32.3\% in males, $15.1 \%$ in females; $p=0,003$ ); MDs12,2\%, (17.1\% in males; $10.2 \%$ in females, $p=0,049), p<0.001$. Among all courses, regular smoking overlaps with college admission; most smokers reported low dependence and desire 
to quit, while $20 \%$ reported readiness to quit; motivation to quit was not triggered by being a role model; Nursing students reported significantly higher dual consumption and experimentation of multiple tobacco products, $p<0.001$. Being female or an MD predicted not using tobacco. Exposure to SHS was rather common in leisure spaces and did not significantly varied among courses.

Conclusions: Tobacco use, experimentation of e-cigarette, and exposure to SHS are common among health science students, highlighting the need to implement comprehensive tobacco control policies in health science schools.

This study was commissioned and funded by the National Tobacco Control Program, Directorate of Health, Portugal. Results and interpretations expressed in this study do not necessarily reflect the opinions of the Directorate of Health. Aknowledgments: To Professor Emília Nunes and the National Tobacco Control Program team for outstanding support. 